\title{
Periodic Solutions of Vibrating Strings. Degree Theory Approach $\left({ }^{*}\right)$.
}

\author{
SŁAWOMIR RYBICKI
}

\begin{abstract}
The aim of this article is to study bifurcations and continuation of T-periodic solutions of a family of string equations. As the main tool we use the global Lyapunov-Schmidt reduction and degree theory for $S^{1}$-equivariant gradient maps defined in [23].
\end{abstract}

\section{1. - Introduction.}

$T$-periodic solutions of a string equation

$$
\begin{cases}u_{t t}-u_{x x}=f(t, x, u) & \text { in }(0, \pi) \times R \\ u(0, t)=u(\pi, t)=0 & \text { for all } t \in R\end{cases}
$$

have been studied among the others in [6]-[15], [18], [21], [22] and [31]. The authors have proved the existence of $T$-periodic solutions of equation (1.1) and multiplicity results for this equation.

On the other hand there are few articles, see for instance [19], [20], [30], in which the authors study bifurcations and continuation of $T$-periodic solutions of the following 1-parameter family of string equations

$$
\begin{cases}u_{t t}-u_{x x}=f(x, u, \lambda) & \text { in }(0, \pi) \times R \\ u(0, t)=u(\pi, t)=0 & \text { for all } t \in R .\end{cases}
$$

The aim of this article is to study such phenomena as bifurcations and continuation of $T$ periodic solutions of family (1.2). Usually only the local bifurcations are considered for periodic solutions of family (1.2), which are guaranteed by the variational methods i.e. Conley index (see [7]), Morse theory (see [13], [31]), cohomological index theory (see [16]) and their equivariant versions. Nontriviality of bifurcation index, defined in terms of classical invariants, does not imply continuation and global bifurcation of critical points of functionals, see famous examples by Böhme [9] and Takens [29]. The only mathematical tool, known to the author, which is suitable for the study of continuation and global bifurcations of critical

(*) Entrata in Redazione il 19 dicembre 1998. Ricevuto nuova versione il 27 maggio 1999

Indirizzo degli AA.: Faculty of Mathematics and Informatics, Nicholas Copernicus University, PL87-100 Toruń, ul. Chopina 12/18, Poland. E-mail: rybicki@mat.uni.torun.pl

Supported by Nicholas Copernicus University grant $341 \mathrm{M}$. 
points of symmetric functional is degree for $S^{1}$-equivariant gradient maps. In fact the author do not know any global bifurcation theorems describing connected components of the set of nontrivial solutions for family (1.2) which bifurcate from the set of trivial solutions or from infinity. We will touch only a few aspects of global bifurcations (from the set of trivial solutions and from infinity) and continuation of $T$-periodic solutions of family (1.2). We emphasize that we can obtain analogous results replacing condition " $0<\alpha<\beta$ " in assumption (F.2) with condition " $\alpha<\beta<0$ ".

The basic idea is to apply both the global Lyapunov-Schmidt reduction, see [2]-[6], and the degree theory for $S^{1}$-equivariant gradient maps constructed by the author in [23], developed in [24] and applied to elliptic differential equations and to Hamiltonian systems in [25]-[28]. The advantage of using degree theory for $S^{1}$-equivariant gradient maps lies in the fact that we prove the existence of continua of $T$-periodic solutions (closed connected sets) which are not given by the variational methods. The disadvantage is that our degree is not capable of recovering the multiplicity results given by Amann and Zehnder, see [6], by comparing degrees at 0 and at $\infty$. On the other hand we discuss in [28] an example of asymptotically linear Hamiltonian system, see Example 4.5 of [28], such that Amann and Zehnder results are not applicable to this system but our degree theory still guarantees the existence of at least one nonstationary periodic solution of this system. It is worth to point out that the degree theory for $G$-equivariant gradient maps ( $G$ is any compact Lie group) has been defined by Gȩba in [17].

The plan of the paper is as follows.

In Section 2 we introduce family (2.1) of autonomous wave equations, set up terminology and notation. We describe eigenspaces of d'Alambertian $\square=u_{t t}-u_{x x}$ as representations of the group $S^{1}$, see formula (2.4). In Lemmas 2.4, 2.7, 2.8 we present the results of global Lyapunov-Schmidt reduction applied to family (2.1). The definition of a bifurcation index is reminded in (2.5).

In Section 3 we study global bifurcations of $T$-periodic solutions of family (2.1) from the set of trivial solutions. We begin this section with two global bifurcation theorems, Theorem 3.1 and 3.2. In Corollaries 3.3, 3.5 we have formulated conditions which imply the existence of continua of $T$-periodic solutions of family (2.1) which bifurcate from the set of trivial solutions. The isotropy groups of $T$-periodic solutions of family (2.1) are studied in Corollaries 3.7, 3.8. We finish this section with Remark 3.9 on standing waves and local bifurcation theory.

Section 4 deals with the case of bifurcations of $T$-periodic solutions of family (2.1) from infinity. In Theorems 4.1, 4.2 and Corollary 4.3 we describe continua of $T$-periodic solutions of family (2.1) of a large norm.

Section 5 is devoted to the study of continuation of $T$-periodic solutions of family (2.1). In Theorem 5.1 we consider family (2.1) without resonance at the origin and at infinity. In Theorems 5.2, 5.5 we study the continuation of $T$-periodic solutions in the presence of resonance at the origin. It is possible that family (2.1) has resonance at infinity but we will not develop this point here.

For the convenience of the reader, in Section 6 , we present without proofs some bifurcation and continuation theorems for $S^{1}$-equivariant gradient maps, thus making our exposition self-contained. 


\section{2. - Preliminaria.}

The aim of this section is to set up terminology and notation. Let us consider 1-parameter family of autonomous wave equations of the form

$$
\begin{cases}u_{t t}-u_{x x}=f(x, u, \lambda) & \text { in }(0, \pi) \times R \\ u(0, t)=u(\pi, t)=0 & \text { for all } t \in R \\ u(x, t)=u(x, t+T) & \text { for all }(x, t) \in(0, \pi) \times R .\end{cases}
$$

Throughout this article we will assume that family (2.1) satisfies the following assumptions (F.1) $f \in C^{2}([0, \pi] \times R \times R, R)$,

(F.2) there exist constants $0<\alpha<\beta$ such that

$$
\alpha \leqslant f_{\xi}^{\prime}(x, \xi, \lambda) \leqslant \beta
$$

for all $(x, \xi, \lambda) \in[0, \pi] \times R \times R$,

(F.3) $\tau=(2 \pi / T) \in Q$.

We let $\Omega=(0, \pi) \times(0, T)$ and $H=L^{2}(\Omega)$, and define a linear operator $\square: \operatorname{dom}(\square) \subset H \rightarrow H$ by

$$
\square u:=u_{t t}-u_{x x}
$$

where $\quad \operatorname{dom}(\square)=\left\{u \in C^{2}(d(\Omega)): u(0, \cdot)=u(\pi, \cdot), u(\cdot, 0)=u(\cdot, T), u_{t}(\cdot, 0)=u_{t}(\cdot, T)\right\}$. Then it is known that $A:=\square^{*}$, the adjoint of $\square$, is self-adjoint and has a pure point spectrum, given by

$$
\left.\sigma(A):=\left\{j^{2}-\tau^{2} \cdot k^{2}:(j, k) \in N^{*} \times Z\right)\right\},
$$

where $N^{*}=\{1,2,3, \ldots\}$. It is easily verified that each $\mu \in \sigma(A)-\{0\}$ is an eigenvalue of finite multiplicity, whereas $\mu_{0}=0$ is an eigenvalue of infinite multiplicity.

More precisely, letting

$$
\begin{gathered}
\varphi_{j}(x):=\sqrt{\frac{2}{\pi} \cdot \sin (\tau \cdot j \cdot x) \quad j \in N^{*},} x \in[0, \pi] \\
\psi_{k}(t)= \begin{cases}\sqrt{\frac{2}{T}} \cdot \cos (\tau \cdot k \cdot t) & \text { for } k \geqslant 1 \\
\sqrt{\frac{1}{T}} & \text { for } k=0 \\
\sqrt{\frac{2}{T} \cdot \sin (\tau \cdot k \cdot t)} & \text { for } k \leqslant-1\end{cases}
\end{gathered}
$$

and all $t \in[0, T]$, it is easily verified that, for each $\mu \in \sigma(A)$, the family

$$
\left\{\varphi_{j}(x) \cdot \psi_{k}(t):(j, k) \in N^{*} \times Z \text { and } j^{2}-\tau^{2} \cdot k^{2}=\mu\right\}
$$

forms an orthonormal basis for the eigenspace $\operatorname{ker}(A-\mu)$ of $A$. In the following we denote by $F$ the Nemyts'kii operator of the function $f$, that is, for every $u: \Omega \rightarrow R$ and 
$\lambda \in R$, the function $F(u, \lambda): \Omega \rightarrow R$ is defined by

$$
F(u, \lambda)(x, t)=f(x, u(x, t), \lambda) \quad \text { for all }(x, t) \in \Omega .
$$

It is known that $F \in C(H \times R, H)$.

Let $S^{1}=\{z \in C:|z|=1\}$ denote the group of complex numbers of module one with an action given by the multiplication of complex numbers and let

$$
Z_{k}=\left\{g \in S^{1}: g=e^{\sqrt{-1} \cdot \theta} \text { for } q=\frac{2 \cdot \pi \cdot j}{k} \text { and } j=0,1, \ldots, k-1\right\}
$$

be a cyclic group. Since family (2.1) is autonomous, in order to research the solution-set of this system, we will use topological invariants with $S^{1}$-symmetries. Let $V$ denote a finite-dimensional real representation of the group $S^{1}$. A group $S_{v}^{1}=\left\{g \in S^{1}: g \cdot v=v\right\}$ will be called the isotropy group of $v \in V$. Moreover, let $\langle\cdot, \cdot\rangle$ be an $S^{1}$-invariant inner product in orthogonal representation $V$ of $S^{1}$. If $V_{0} \subset V$ is a subrepresentation of $V$ then

$$
V_{0}^{\perp}=\left\{v \in V:\left\langle v, v_{0}\right\rangle=0 \forall v_{0} \in V_{0}\right\} \quad \text { and } \quad V^{Z_{k}}=\left\{v \in V: g \cdot v=v \forall g \in Z_{k}\right\} .
$$

For $k \in N^{*}$ define a map $\varrho^{k}: S^{1} \rightarrow G L(2, R)$ as follows

$$
\varrho^{k}\left(e^{\sqrt{-1} \cdot \theta}\right)=\left[\begin{array}{cc}
\cos k \cdot \theta & -\sin k \cdot \theta \\
\sin k \cdot \theta & \cos k \cdot \theta
\end{array}\right] \quad 0 \leqslant \theta<2 \cdot \pi .
$$

For $j, k \in N$ we denote by $R[j, k]$ the direct sum of $j$ copies of $\left(R^{2}, \varrho^{k}\right)$, we also denote by $R[j, 0]$ the trivial $j$-dimensional representation of $S^{1}$. We say that two representations $V$ and $W$ are equivalent if there exists an equivariant, linear isomorphism $T: V \rightarrow W$. The following classic result gives a complete classification (up to equivalence) of finite-dimensional representations of the group $S^{1}$ (see [1]).

THEOREM 2.1 (Classification theorem). - If $V$ is a representation of $S^{1}$ then there exist finite sequences $\left\{j_{i}\right\},\left\{k_{i}\right\}$ satisfying

$$
k_{i} \in\{0\} \cup N, \quad j_{i} \in N, \quad 1 \leqslant i \leqslant r, \quad k_{1}<k_{2}<\ldots<k_{r}
$$

such that $V$ is equivalent to $\bigoplus_{i=1}^{r} R\left[j_{i}, k_{i}\right]$.

Moreover, the equivalence class of $V\left(V \approx \bigoplus_{i=1}^{\prime} R\left[j_{i}, k_{i}\right]\right)$ is uniquely determined by $\left\{j_{i}\right\}$, $\left\{k_{i}\right\}$ satisfying $(*)$.

DEFINITION 2.2. - Let $V \approx \bigoplus_{i=1}^{r} R\left[j_{i}, k_{i}\right]$. Subgroup $Z_{k} \subset S^{1}$ is said to be a maximal isotropy subgroup in $V$ if $k \in\left\{k_{1}, \ldots, k_{r}\right\}$ and $k_{i} / k \notin N$ for any $k_{i} \neq k$.

Let us introduce an action of the group $S^{1}$ on $H$ in the following way

$$
e^{\sqrt{-1} \varphi} \cdot u(x, t)=u\left(x, t+\frac{\varphi}{\tau}\right)
$$

for $\varphi \in[0,2 \pi)$ and $u \in H$ (see [6] for more details). Fix $\mu \in \sigma(A)-\{0\}$. It is evident that 
$\operatorname{ker}(A-\mu)$ is a finite-dimensional representation of the group $S^{1}$. Notice that

$$
\begin{aligned}
& \left\{(j, k) \in N^{*} \times Z: j^{2}-\tau^{2} k^{2}=\mu\right\}= \\
& \quad= \begin{cases}\left\{(\sqrt{\mu}, 0),\left(j_{1}, k_{1}\right),\left(j_{1},-k_{1}\right), \ldots\left(j_{r}, k_{r}\right),\left(j_{r},-k_{r}\right)\right\} & \text { if } \sqrt{\mu} \in N^{*} \\
\left\{\left(j_{1}, k_{1}\right),\left(j_{1},-k_{1}\right), \ldots\left(j_{r}, k_{r}\right),\left(j_{r},-k_{r}\right)\right\} & \text { if } \sqrt{\mu} \notin N^{*} .\end{cases}
\end{aligned}
$$

Taking into account the action of the group $S^{1}$ given by (2.2) and (2.3) it is easy to see that

$$
\operatorname{ker}(A-\mu) \approx \begin{cases}R[1,0] \oplus \bigoplus_{i=1}^{r} R\left[1, k_{i}\right] & \text { if } \sqrt{\mu} \in N^{*} \\ \bigoplus_{i=1}^{r} R\left[1, k_{i}\right] & \text { if } \sqrt{\mu} \notin N^{*}\end{cases}
$$

Family (2.1) satisfies all the assumptions of the global Lyapunov-Schmidt reduction, see [2]-[6]. Thanks to Remark 2.2 in [4] we can apply this reduction in a parameterized version.

Let $\sigma(A) \cap(\alpha, \beta)=\left\{\mu_{1}, \ldots, \mu_{n}\right\}$, where $\mu_{1}<\mu_{2}<\ldots<\mu_{n}$ and let

$$
Z:=\operatorname{ker}\left(A-\mu_{1}\right) \oplus \ldots \oplus \operatorname{ker}\left(A-\mu_{n}\right) .
$$

Observe that $Z$ is a finite-dimensional representation of the group $S^{1}$.

Definition 2.3. - An element $(u, \lambda) \in \operatorname{dom}(A) \times R$ is said to be a weak solution of family (2.1), if $A(u)=F(u, \lambda)$.

Let $\nabla b(x)$ and $\nabla^{2} b(x)$ denote the gradient and the hessian of a function $b: R^{n} \rightarrow R$, respectively. Assume that function $f$ satisfies the following assumption

(F.4) $f(x, \xi, \lambda)=b(\lambda) \cdot \xi+g(x, \xi, \lambda)$, where $g(x, 0, \lambda)=0$ and $g_{\xi}^{\prime}(x, 0, \lambda)=0$ for all $(x, \lambda) \in[0, \pi] \times R$ and $b: R \rightarrow R$ is a continuous function.

The following lemma is a parameterized version of Lemma 3.1 of [6].

Lemma 2.4. - Assume that family (2.1) satisfies assumptions (F.1)-(F.4). Then there is a parameterized family of functionals $a \in C^{2}(Z \times R, R)$ with the following properties:

(a.1) the critical points of a (with respect to the first coordinate) are in a one-to-one correspondence with weak solutions of family (2.1). More precisely, if $\operatorname{dom}(A)=Z \oplus Z^{\perp}$ then there is a continuous $S^{1}$-equivariant map $v: Z \times R \rightarrow Z^{\perp}$ such that

$$
\nabla a(z, \lambda)=0 \quad \text { iff } A(z+v(z, \lambda))=F(z+v(z, \lambda), \lambda),
$$

(a.2) $\nabla a(0, \lambda)=0$ for all $\lambda \in R$,

(a.3) $a$ is invariant with respect to the action of the group $S^{1}$ defined in (2.2),

(a.4) $\nabla^{2} a(0, \lambda)=A-b(\lambda)$.

A proof of this lemma one can find in [4]. We will study global bifurcations of periodic solutions of system (2.1).

DeFinition 2.5. - An element $\left(0, \lambda_{0}\right) \in \operatorname{dom}(A) \times R$ is said to be a bifurcation point of weak solutions of family (2.1), if there is a sequence $\left\{\left(u_{n}, \lambda_{n}\right)\right\} \subset d o m(A) \times R$ such that 1. $A\left(u_{n}\right)=F\left(u_{n}, \lambda_{n}\right)$ for any $n \in N^{*}$ i.e. for any $n \in N^{*}$ the pair $\left(u_{n}, \lambda_{n}\right)$ is a weak solution of (2.1),

2. $\lim _{n \rightarrow \infty} u_{n}=0$ and $\lim _{n \rightarrow \infty} \lambda_{n}=\lambda_{0}$. 
It is easy to see that if $\left(0, \lambda_{0}\right)$ is a bifurcation point of weak solutions of family (2.1) then $b\left(\lambda_{0}\right) \in\left\{\mu_{1}, \mu_{2}, \ldots, \mu_{n}\right\}$. Let us fix $\lambda_{0} \in R$ such that $b\left(\lambda_{0}\right) \in\left\{\mu_{1}, \mu_{2}, \ldots, \mu_{n}\right\}$ and $\varepsilon>0$ such that $\left(\lambda_{0}-\varepsilon, \lambda_{0}+\varepsilon\right) \cap b^{-1}\left(\left\{\mu_{1}, \mu_{2}, \ldots, \mu_{n}\right\}\right)=\left\{\lambda_{0}\right\}$. From Lemma 2.4 it follows that there is $\alpha>0$ such that $\nabla a(\cdot, \lambda \pm \varepsilon)^{-1}(0) \cap c l\left(D_{a}(Z)\right)=\{0\}$, where $D_{a}(Z)=$ $=\{z \in Z:|z|<\alpha\}$. Taking into consideration the above we are in a position to define a bifurcation index $\eta\left(\lambda_{0}\right)$ at $\lambda_{0} \in R$ in the following way

$$
\eta\left(\lambda_{0}\right)=D E G\left(\nabla a\left(\cdot, \lambda_{0}+\varepsilon\right), D_{\alpha}(Z)\right)-D E G\left(\nabla a\left(\cdot, \lambda_{0}-\varepsilon\right), D_{\alpha}(Z)\right)
$$

where $D E G(\cdot, \cdot) \in Z \oplus\left(\bigoplus_{i=1}^{\infty} Z\right)$ denotes degree for $S^{1}$-equivariant gradient maps defined in [23]. Suppose that $b\left(\lambda_{0} \pm \varepsilon\right) \notin\left\{\mu_{1}, \mu_{2}, \ldots, \mu_{n}\right\}$. In other words $\nabla^{2} a\left(0, \lambda_{0} \pm \varepsilon\right)$ is an isomorphism. Thus repeating reasoning from Lemma 4.1 of [23] we obtain

$$
\eta\left(\lambda_{0}\right)=D E G\left(\nabla^{2} a\left(0, \lambda_{0}+\varepsilon\right), D_{\alpha}(Z)\right)-D E G\left(\nabla^{2} a\left(0, \lambda_{0}-\varepsilon\right), D_{\alpha}(Z)\right) .
$$

And finally by Lemma 2.4 (a.4) we have

$$
\eta\left(\lambda_{0}\right)=D E G\left(A-b\left(\lambda_{0}+\varepsilon\right), D_{\alpha}(Z)\right)-D E G\left(A-b\left(\lambda_{0}-\varepsilon\right), D_{\alpha}(Z)\right) .
$$

Assume that function $f$ satisfies the following assumption

(F.5) $f(x, \xi, \lambda)=b(\lambda) \cdot \xi+g(x, \xi, \lambda)$, where $g(x, \xi, \lambda)=o(\|\xi\|)$, at $\xi=\infty$, uniformly in $x \in[0, \pi]$ and in bounded $\lambda$-intervals.

Definition 2.6. - $A$ gradient $\nabla b$ of a $C^{2}$ function $b: R^{n} \rightarrow R$ is said to be asymptotically linear at $x=\infty$, if there is a symmetric $n \times n$ matrix $\nabla^{2} b(\infty)$ such that $\| \nabla b(x)-$ $-\nabla^{2} b(\infty) x \|=o(\|x\|)$ at $x=\infty$.

Notice that we can relax assumption (E) of Theorem 4 of [12], replacing it with assumption (E') $0 \notin \sigma\left(\left(A-F_{\infty}\right)_{\mid H^{-} \oplus H^{+}}\right)$. Combining Lemma 3.1 of [6] with, proposed above, stronger version of Theorem 4 of [12] we obtain the following lemma.

Lemma 2.7. - Suppose that family (2.1) satisfies assumptions (F.1)-(F.3), (F.5). Then there is a parameterized family of functionals $a \in C^{2}(Z \times R, R)$ with the following properties:

(a.1) the critical points of a (with respect to the first coordinate) are in a one-to-one correspondence with weak solutions of family (2.1). More precisely, if $\operatorname{dom}(A)=Z \oplus Z^{\perp}$ then there is a continuous $S^{1}$-equivariant map $v: Z \times R \rightarrow Z^{\perp}$ such that

$$
\nabla a(z, \lambda)=0 \quad \text { iff } A(z+v(z, \lambda))=F(z+v(z, \lambda), \lambda),
$$

(a.2) $a$ is invariant with respect to the action of the group $S^{1}$ defined in (2.2),

(a.3) $\nabla a(\cdot, \lambda)$ is asymptotically linear at $z=\infty$,

(a.4) $\nabla^{2} a(\infty, \lambda)=A-b(\lambda)$.

In the rest of this section we formulate lemma which will be used in the studies of continuations of periodic solutions of family (2.1). We assume that family (2.1) satisfies the following assumptions: there are $\lambda_{0} \in R$ and $\mu_{0}, \mu_{\infty} \in(\alpha, \beta)$ such that

(F.6) $f\left(x, \xi, \lambda_{0}\right)=\mu_{0} \xi+g_{0}(x, \xi)$, where $g_{0}(x, 0)=\left(g_{0}\right)_{\xi}^{\prime}(x, 0)=0$ for all $x \in[0, \pi]$,

(F.7) $f\left(x, \xi, \lambda_{0}\right)=\mu_{\infty} \xi+g_{\infty}(x, \xi)$, where $g_{\infty}(x, \xi)=o(\|\xi\|)$, at $\xi=\infty$, uniformly in $x \in[0, \pi]$. 
Combining Lemma 3.1 of [6] with Theorem 4 of [12] we obtain the following lemma.

Lemma 2.8. - Suppose that family (2.1) satisfies assumptions (F.1)-(F.3). Then there is a parameterized family of functionals $a \in C^{2}(Z \times R, R)$ with the following properties:

(a.1) the critical points of a (with respect to the first coordinate) are in a one-to-one correspondence with weak solutions of family (2.1). More precisely, if dom $(A)=Z \oplus Z^{\perp}$ then there is a continuous $S^{1}$-equivariant map $v: Z \times R \rightarrow Z^{\perp}$ such that $\nabla a(z, \lambda)=0$ iff $A(z+v(z, \lambda))=F(z+v(z, \lambda), \lambda)$,

(a.2) $a$ is invariant with respect to the action of the group $S^{1}$ defined in (2.2).

If family (2.1) satisfies assumption (F.6) then

(a.3) $\nabla a\left(0, \lambda_{0}\right)=0$,

(a.4) $\nabla^{2} a\left(0, \lambda_{0}\right)=A-\mu_{0}$.

If family (2.1) satisfies assumption (F.7) then

(a.5) $\nabla a\left(\cdot, \lambda_{0}\right)$ is asymptotically linear at $z=\infty$,

(a.6) $\nabla^{2} a\left(\infty, \lambda_{0}\right)=A-\mu_{\infty}$.

\section{3. - Global bifurcations of periodic solutions.}

In this section we prove two kinds of results. Namely, we prove global bifurcation theorems describing the global properties of the set of solutions of family (2.1) and symmetrybreaking results. Throughout this section we assume that family (2.1) satisfies assumptions (F.1)-(F.4).

Let us denote by $C\left(\lambda_{0}\right)$ connected component of the set

$$
c l\{(u, \lambda) \in \operatorname{dom}(A) \times R: A u=F(u, \lambda) \text { and } u \neq 0\}
$$

such that $\left(0, \lambda_{0}\right) \in C\left(\lambda_{0}\right)$. This theorem yields information about behaviour of $C\left(\lambda_{0}\right)$.

THEOREM 3.1. - Fix $\lambda_{0} \in R$ such that $b\left(\lambda_{0}\right) \in\left\{\mu_{1}, \ldots, \mu_{n}\right\}$ and assume that function $b(\lambda)-b\left(\lambda_{0}\right)$ changes sign at $\lambda_{0}$. Then $\left(0, \lambda_{0}\right) \in \operatorname{dom}(A) \times R$ is a bifurcation point of weak solutions of family (2.1). Moreover, $C\left(\lambda_{0}\right)$ is either unbounded in $\operatorname{dom}(A) \times R$ or $\left(C\left(\lambda_{0}\right)-\left(0, \lambda_{0}\right)\right) \cap\left(\{0\} \times b^{-1}\left(\left\{\mu_{1}, \ldots, \mu_{n}\right\}\right)\right) \neq \emptyset$.

PROOF. - We first observe that Lemma 2.1 (a.1) shows that in order to study weak solutions of family (2.1) it is enough to deal with zeros of an $S^{1}$-equivariant gradient map $\nabla a: Z \times R \rightarrow Z$. Since any eigenspace $\operatorname{ker}\left(A-\mu_{k}\right)$ is nontrivial or odd-dimensional representation of the group $S^{1}$ and $h(\lambda)-b\left(\lambda_{0}\right)$ changes sign at $\lambda_{0}$ by (2.6) and Lemma 4.3 of [23] we have $\eta\left(\lambda_{0}\right) \neq \Theta \in Z \oplus\left(\bigoplus_{i=1}^{\infty} Z\right)$. The rest of our proof is a consequence of Lemma 2.4 and Theorem 6.1.

The number of elements of the set $S$ will be denoted by \#S. Loosely speaking, the formula in the following theorem says that the sum of bifurcation indices, which corresponds to 
the bounded component $C\left(\lambda_{0}\right)$, is trivial in $Z \oplus\left(\bigoplus_{i=1}^{\infty} Z\right)$. The following theorem will prove extremely useful in the second part of this section. Applying this theorem we will exclude the existence of bounded components of nontrivial solutions of family (2.1) which bifurcate from the set of trivial solutions.

THEOREM 3.2. - Fix $\lambda_{0} \in R$ such that $b\left(\lambda_{0}\right) \in\left\{\mu_{1}, \ldots, \mu_{n}\right\}$ and assume that function $b(\lambda)-b\left(\lambda_{0}\right)$ changes sign at $\lambda_{0}$. Then $\left(0, \lambda_{0}\right) \in \operatorname{dom}(A) \times R$ is a bifurcation point of weak solutions of family (2.1). Moreover, if \# $\left(b^{-1}\left(\left\{\mu_{1}, \ldots, \mu_{n}\right\}\right) \cap\left[\gamma_{1}, \gamma_{2}\right]\right)<\infty$ for any interval $\left[\gamma_{1}, \gamma_{2}\right] \subset R$ then $C\left(\lambda_{0}\right)$ is either unbounded in dom $(A) \times R$ or $\mathbf{C}\left(\lambda_{0}\right) \cap(\{0\} \times R)=$ $=\{0\} \times\left\{\lambda_{i_{1}}, \ldots, \lambda_{i_{s}}\right\} \subset\{0\} \times h^{-1}\left(\left\{\mu_{1}, \ldots, \mu_{n}\right\}\right)$ and

$$
\sum_{j=1}^{s} \eta\left(\lambda_{i_{j}}\right)=\Theta \in Z \oplus\left(\bigoplus_{i=1}^{\infty} Z\right)
$$

PROOF. - The proof of this theorem is literally the same as the proof of Theorem 3.1. But in the proof we should apply Theorem 6.2 instead of Theorem 6.1.

There are many conditions which exclude the existence of bounded components of nontrivial solutions of family (2.1). The point of the following corollaries is to present some of them.

Corollary 3.3. - If

a) function $b: R \rightarrow R$ is increasing in a neigbbourbood of any point of the set $b^{-1}\left\{\mu_{1}, \ldots, \mu_{n}\right\}$ and $\left\{\mu_{1}, \ldots, \mu_{n}\right\} \cap\left\{\mu^{2}: \mu \in N^{*}\right\}=\emptyset$ or

b) function $b: R \rightarrow R$ is increasing in a neighbourbood of any point of the set $b^{-1}\left\{\mu_{1}, \ldots, \mu_{n}\right\}$ and for any $\mu_{i} \in\left\{\mu_{1}, \ldots, \mu_{n}\right\}$ there is a subrepresentation $R\left[1, k_{i}\right]$ of $\operatorname{ker}\left(A-\mu_{i}\right)$ which is not a subrepresentation of $\operatorname{ker}\left(A-\mu_{j}\right)$ for any $\mu_{i} \neq \mu_{j}$ or

then any continuum of nontrivial solutions of family (2.1) which bifurcate from the set of trivial solutions is unbounded in $\operatorname{dom}(A) \times R$.

ProOF. - The basic idea of the proof is to show that the sum of any finite number of bifurcation indices which appear in our situation is nontrivial in $Z \oplus\left(\bigoplus_{i=1}^{\infty} Z\right)$. In other words we will exclude one of possibilities of behaviour of $C\left(\lambda_{0}\right)$ which occur in Theorem 3.2.

a) Our proof starts with the observation that from (2.4) and assumptions it follows that $\operatorname{ker}\left(A-\mu_{i}\right)$ is an even-dimensional representation of the group $S^{1}$ such that $\operatorname{ker}(A-$ $\left.-\mu_{i}\right)^{S^{1}}=\{0\}$, for any $\mu_{i} \in\left\{\mu_{1}, \ldots, \mu_{n}\right\}$. Fix $\lambda_{0} \in b^{-1}\left\{\mu_{1}, \ldots, \mu_{n}\right\}$. Taking into account that $b$ is increasing and combining (2.6) with Lemma 4.3 of [23] we show that the bifurcation index $\eta\left(\lambda_{0}\right)$ is nontrivial and that any nonzero coordinate of this index is positive. The rest of our proof is a direct consequence of Theorem 3.2.

b) Fix $\lambda_{0} \in b^{-1}\left\{\mu_{1}, \ldots, \mu_{n}\right\}$. Since for any $\mu_{j} \in\left\{\mu_{1}, \ldots, \mu_{n}\right\} \# b^{-1}\left(\mu_{j}\right)=0$ or 1 there is $\mu_{i}$ such that $\lambda_{0}=b^{-1}\left(\mu_{i}\right)$. Since $b$ is increasing the coordinate $\eta\left(\lambda_{0}\right)_{z_{k_{i}}}$ of bifurcation index $\eta\left(\lambda_{0}\right)$ which corresponds to isotropy subgroup $Z_{k_{i}}$ is equal to 1 . Taking into con- 
sideration assumptions and Lemma 4.3 of [23] we can show that if $\lambda_{1} \in b^{-1}\left(\left\{\mu_{1}, \ldots, \mu_{n}\right\}-\left\{\mu_{i}\right\}\right)$ then $\eta\left(\lambda_{1}\right)_{z_{i}}=0$. The rest of our proof is a direct consequence of Theorem 3.2.

REMARK 3.4. - Under the assumptions of Corollary 3.3 with «increasing» replaced by «decreasing» we still obtain the thesis of this corollary.

The interest of the following corollary is that it allows one to prove the existence of unbounded components of nontrivial solutions of family (2.1) even if not all of them are unbounded.

CoROLlaRY 3.5. - a) Fix $k \in N^{\star}$. If $\# \mathfrak{G}(k)=\#\left\{\lambda_{0} \in b^{-1}\left\{\mu_{1}, \ldots, \mu_{n}\right\}\right.$ such that $R[1, k]$ is a subrepresentation of ker $\left.\left(A-b\left(\lambda_{0}\right)\right)\right\}$ is odd and function $b(\lambda)$ is increasing or decreasing in a neigbbourbood of any point of the set $\mathfrak{A}(k)$ then there is $\lambda_{0} \in \mathfrak{G}(k)$ such that $C\left(\lambda_{0}\right)$ is unbounded in $\operatorname{dom}(A) \times R$. b) If $\#\left(b^{-1}\left(\left\{\mu_{1}, \ldots, \mu_{n}\right\}\right) \cap\left[\gamma_{1}, \gamma_{2}\right]\right)<\infty$ for any interval $\left[\gamma_{1}, \gamma_{2}\right] \subset R$ and there is $\mu_{i}$ such that $\# b^{-1}\left(\mu_{i}\right)$ is odd, $R[1, k]$ is a subrepresentation of $\operatorname{ker}\left(A-\mu_{i}\right), R[1, k]$ is not a subrepresentation of $\operatorname{ker}\left(A-\mu_{j}\right)$ for any $\mu_{j} \neq \mu_{i}$ and function $b(\lambda)$ is increasing or decreasing in a neighbourbood of any point of the set $b^{-1}\left\{\mu_{i}\right\}$ then there is $\lambda_{0} \in b^{-1}\left\{\mu_{i}\right\}$ such that $C\left(\lambda_{0}\right)$ is unbounded in $\operatorname{dom}(A) \times R$.

PROOF. - The main idea of the proof is to show that only the sum of even number of bifurcation indices which appear in our situation can be the trivial element in $Z \oplus\left(\bigoplus_{i=1}^{\infty} Z\right)$.

a) Fix $\lambda_{0} \in \mathcal{Q}(k)$. By the assumption, (2.6) and Lemma 4.3 of [23] we have $\eta\left(\lambda_{0}\right)_{Z_{k}}=$ $= \pm 1$. Hence only the sum of an even number of bifurcation indices (computed at points of $\mathfrak{A}(k))$ can be equal to the trivial element in $Z \oplus\left(\oplus_{i=1}^{\infty} Z\right)$. Thus applying Theorem 3.2 we conclude that if $C\left(\lambda_{0}\right)$ is bounded in $\operatorname{dom}(A) \times R$ then $\#\left(C\left(\lambda_{0}\right) \cap(\{0\} \times\right.$ $\times \mathfrak{A}(k)))$ is even. Suppose, contrary to our claim, that for any $\lambda_{0} \in \mathfrak{A}(k) \mathcal{C}\left(\lambda_{0}\right)$ is bounded in $\operatorname{dom}(A) \times R$. Since $\# \mathfrak{Q}(k)<\infty$ is finite we prove that $\# \mathfrak{G}(k)$ is even, which contradicts the assumption.

b) Fix $\lambda_{0} \in b^{-1}\left\{\mu_{1}, \ldots, \mu_{n}\right\}$. By the assumption, (2.6) and Lemma 4.3 of [23] we have

$$
\eta\left(\lambda_{0}\right)_{Z_{k}}= \begin{cases} \pm 1 & \text { if } \lambda_{0} \in b^{-1}\left\{\mu_{i}\right\} \\ 0 & \text { otherwise }\end{cases}
$$

Hence only the sum of even number of bifurcation indices, which corresponds to $b^{-1}\left(\mu_{i}\right)$, can be equal to the trivial element in $Z \oplus\left(\bigoplus_{i=1}^{\infty} Z\right)$. Thus applying Theorem 3.2 we conclude that if $\lambda_{0} \in b^{-1}\left(\mu_{i}\right)$ and $C\left(\lambda_{0}\right)$ is bounded in $\operatorname{dom}(A) \times R$ then $\#\left(C\left(\lambda_{0}\right) \cap(\{0\} \times\right.$ $\left.\left.\times b^{-1}\left(\mu_{i}\right)\right)\right)$ is even. Suppose, contrary to our claim, that for any $\lambda_{0} \in b^{-1}\left\{\mu_{i}\right\} C\left(\lambda_{0}\right)$ is bounded in $\operatorname{dom}(A) \times R$. Since $b^{-1}\left\{\mu_{i}\right\}$ is finite we prove that $\# b^{-1}\left\{\mu_{i}\right\}$ is even, which contradicts the assumption.

EXAMPLE 3.6. - In order to illustrate Corollary 3.5 a we present bere the following example. Let us consider family (2.1) satisfying all the assumptions of Corollary 3.5 with:

$$
4<\alpha<\mu_{1}=5<\mu_{2}=7<\mu_{3}=8<\mu_{4}=9<\mu_{5}=11<\mu_{6}=12<\beta<13, \quad T=2 \pi \text {. }
$$


It is clear that $\sigma(A) \cap(\alpha, \beta)=\{5,7,8,9,11,12\}, \operatorname{ker}(A-5) \approx R[1,2], \operatorname{ker}(A-7) \approx$ $\approx R[1,3], \quad \operatorname{ker}(A-8) \approx R[1,1], \quad \operatorname{ker}(A-9) \approx R[1,0] \oplus R[1,4], \quad \operatorname{ker}(A-11) \approx$ $\approx R[1,5], \quad \operatorname{ker}(A-12) \approx R[1,2] . \quad$ Letting $k=2$ assume that $\# \mathfrak{Q}(2)=$ $=\#\left(b^{-1}(5) \cup b^{-1}(12)\right)$ is odd. Applying Corollary 3.5 a we obtain that at least one of continua bifurcating from $\mathfrak{a}(2)$ is unbounded. On the other band it can bappen that some continua bifurcating from $b^{-1}\left\{\mu_{1}, \mu_{2}, \mu_{3}, \mu_{4}, \mu_{5}, \mu_{6}\right\}$ are bounded.

The principal significance of the following two corollaries is that they allow one to control isotropy groups of nontrivial solutions of family (2.1). In other words we prove symmetry-breaking of periodic solutions of family (2.1).

COROLLARY 3.7. - Under the bypotheses of Theorem 3.1 if moreover $Z_{k}$ is a maximal isotropy group in ker $\left(A-b\left(\lambda_{0}\right)\right)$ then there is an open neighbourbood of $\Omega \subset \operatorname{dom}(A) \times R$ of $\left(0, \lambda_{0}\right) \in \operatorname{dom}(A) \times R$ such that $S_{(u, \lambda)}^{1}=Z_{k}$ for any $(u, \lambda) \in\left(C\left(\lambda_{0}\right)^{Z_{k}} \cap \Omega\right)-(\{0\} \times R)$.

Proof. - First we restrict the map $\nabla a: Z \times R \rightarrow Z$ to the set of fixed points of the action of the group $Z_{k}$ and consider a map $(\nabla a)^{Z_{k}}: Z^{Z_{k}} \times R \rightarrow Z^{Z_{k}}$. Next we apply Theorem 3.1 to $(\nabla a)^{Z_{k}}$. Finally taking into account that $\operatorname{ker}\left(A-b\left(\lambda_{0}\right)\right)^{Z_{k}} \approx R[1, k]$ and applying $S^{1}$-equivariant and variational Lyapunov-Schmidt reduction at $\left(0, \lambda_{0}\right)$ we show that the isotropy group of any nontrivial solution of the equation $(\nabla a)^{Z_{k}}(z, \lambda)=0$ in a small neighbourhood $\Omega \subset Z^{Z_{k}} \times R$ of $\left(0, \lambda_{0}\right) \in Z^{Z_{k}} \times R$ is equal to $Z_{k}$. What is left is to show that $C\left(\lambda_{0}\right)$ has the same properties as $C\left(\lambda_{0}\right)$. Let us define a map $u: Z \times R \rightarrow \operatorname{dom}(A)=Z \oplus Z^{\perp}$ by the formula $u(z, \lambda)=(z, v(z, \lambda))$, where $v$ is given in Lemma 2.4 (a.1). By Lemma 2.4 we have

1. $(z, \lambda)$ is a solution of the equation $\nabla a(z, \lambda)=0$ iff $u(z, \lambda)$ is a solution of the equation $A(u)=F(u, \lambda)$,

2. since $S_{(z, \lambda)}^{1} \subset S_{u(z, \lambda)}^{1}=S_{(z, \lambda)}^{1} \cap S_{v(z, \lambda)}^{1} \subset S_{(z, \lambda)}^{1}$ we obtain $S_{(z, \lambda)}^{1}=S_{u(z, \lambda)}^{1}$.

In other words $u\left(C\left(\lambda_{0}\right)\right)=C\left(\lambda_{0}\right)$ and corresponding points have the same isotropy subgroups, which completes our proof.

CoRollaRY 3.8. - Under the hypotheses of Theorem 3.1 if moreover

1. $\left\{\mu_{1}, \ldots, \mu_{n}\right\} \cap\left\{\mu^{2}: \mu \in N^{*}\right\}=\emptyset$,

2. $Z_{k}$ is a maximal isotropy subgroup in $Z$ and in $\operatorname{ker}\left(A-b\left(\lambda_{0}\right)\right)$ then $S_{(u, \lambda)}^{1}=Z_{k}$ for any $(u, \lambda) \in C\left(\lambda_{0}\right)^{Z_{k}}-(\{0\} \times R)$.

Proof. - We first observe that $Z^{Z_{k}} \approx R[j, k]$, next notice that $\eta\left(\lambda_{0}\right) \neq \Theta$ and finally repeat the second part of the proof of Corollary 3.7

REMARK 3.9. - Assumption (F.2) is the assumption which allows one to apply the global Lyapunov-Schmidt reduction to family (2.1) and investigate this family from the global bifurcation theory point of view. Suppose that family (2.1) satisfies only assumptions (F.1), (F.3) and (F.4). In this situation we can apply local $S^{1}$-equivariant and gradient Lyapunov-Schmidt reduction to family (2.1) and research the local bifurcations of periodic solutions of family 
(2.1). Notice that if $b\left(\lambda_{0}\right) \in\left\{\mu^{2}: \mu \in N^{*}\right\}$ then Theorems 3.1, 3.2 bold true for standing waves i.e. for solutions which does not depend on time.

\section{4. - Bifurcations from infinity.}

In this section we study bifurcations from infinity of periodic solutions of family $(2,1)$. Throughout this section we assume that family (2.1) satisfies assumptions (F.1)-(F.3), (F.5). Set $\mathscr{F}=\{(u, \lambda) \in \operatorname{dom}(A) \times R: A(u)=F(u, \lambda)\}$. The following two theorems are analogous to theorems for elliptic differential equations proved in [26]. They yields information about periodic solutions of family (2.1) of a large norm.

THEOREM 4.1. - Assume that there is $\lambda_{0} \in R$ such that $b\left(\lambda_{0}\right) \in\left\{\mu_{1}, \ldots, \mu_{n}\right\}$ and $b(\lambda)-$ $-b\left(\lambda_{0}\right)$ changes sign at $\lambda_{0}$. Then $\mathscr{T}$ possesses an unbounded connected component $D\left(\lambda_{0}\right)$ which meets $\left(\infty, \lambda_{0}\right)$ (we add the points $\{(\infty, \lambda): \lambda \in R\}$ to $\left.\operatorname{dom}(A) \times R\right)$. To be more precise, if $\varepsilon_{1}>0$ is such that $\left[\lambda_{0}-\varepsilon_{1}, \lambda_{0}+\varepsilon_{1}\right] \cap h^{-1}\left(\left\{\mu_{1}, \ldots, \mu_{n}\right\}\right)=\left\{\lambda_{0}\right\}$ then for sufficiently large $\varepsilon_{2}>0$ and $M=\left\{u \in \operatorname{dom}(A):\|u\|>\varepsilon_{2}\right\} \times\left(\lambda_{0}-\varepsilon_{1}, \lambda_{0}+\varepsilon_{1}\right)$ either a) $D\left(\lambda_{0}\right)-M$ is bounded in dom $(A) \times R$, in which case $D\left(\lambda_{0}\right)-M$ meets $\{0\} \times\left(Z^{\perp}\right)^{S^{1}} \times R C$ dom $(A) \times R$, or b) $D\left(\lambda_{0}\right)-M$ is unbounded in dom $(A) \times R$. If $\left.\mathrm{b}\right)$ occurs and $D\left(\lambda_{0}\right)-M$ bas a bounded projection on $R$, then $D\left(\lambda_{0}\right)-M$ meets $\left(\infty, \lambda_{0}^{\prime}\right)$, where $\lambda_{0} \neq \lambda_{0}^{\prime}$ and $b\left(\lambda_{0}^{\prime}\right) \in\left\{\mu_{1}, \ldots, \mu_{n}\right\}$.

ProOF We first observe that applying Lemma 2.7 we reduce the study of periodic solutions of family (2.1) to a finite-dimensional problem. Next we apply Theorem 6.3 and finally define $D\left(\lambda_{0}\right)=\left\{(z, v(z, \lambda), \lambda):(z, \lambda) \in \mathscr{O}\left(\lambda_{0}\right)\right\}$.

\section{Theorem 4.2. - Assume that}

1. $\#\left\{\lambda \in\left[\lambda_{1}, \lambda_{2}\right]: b(\lambda) \in\left\{\mu_{1}, \ldots, \mu_{n}\right\}\right\}<\infty$ for any $\lambda_{1}, \lambda_{2} \in R$,

2. there is $\lambda_{0} \in b^{-1}\left\{\mu_{1}, \ldots, \mu_{n}\right\}$ such that $h(\lambda)-b\left(\lambda_{0}\right)$ changes sign at $\lambda_{0}$.

Then $\mathscr{F}$ possesses an unbounded connected component $D\left(\lambda_{0}\right)$ which meets $\left(\infty, \lambda_{0}\right)$ (we add the points $\{(\infty, \lambda): \lambda \in R\}$ to dom $(A) \times R$ ). To be more precise, if $\varepsilon_{1}>0$ is such that $\left[\lambda_{0}-\varepsilon_{1}, \lambda_{0}+\varepsilon_{1}\right] \cap b^{-1}\left(\left\{\mu_{1}, \ldots, \mu_{n}\right\}\right)=\left\{\lambda_{0}\right\}$ then for sufficiently large $\varepsilon_{2}>0$ and $M=$ $=\left\{u \in \operatorname{dom}(A):\|u\|>\varepsilon_{2}\right\} \times\left(\lambda_{0}-\varepsilon_{1}, \lambda_{0}+\varepsilon_{1}\right)$ either $\left.\mathrm{a}\right) D\left(\lambda_{0}\right)-M$ is bounded indom $(A) \times$ $\times R$, in which case $D\left(\lambda_{0}\right)-M$ meets $\{0\} \times\left(Z^{\perp}\right)^{S^{1}} \times R \subset \operatorname{dom}(A) \times R$, or b) $D\left(\lambda_{0}\right)-M$ is unbounded in dom $(A) \times R$. If $\mathrm{b})$ occurs and $D\left(\lambda_{0}\right)-M$ bas a bounded projection on $R$, then $D\left(\lambda_{0}\right)-M$ meets $\left(\infty, \lambda_{0}^{\prime}\right)$, where $\lambda_{0} \neq \lambda_{0}^{\prime}$ and $h\left(\lambda_{0}^{\prime}\right) \in\left\{\mu_{1}, \ldots, \mu_{n}\right\}$. Additionally, if $D\left(\lambda_{0}\right) \cap(\{0\} \times R)=\emptyset$, then

$$
D\left(\lambda_{0}\right) \cap\{(\infty, \lambda): \lambda \in R\}=\left\{\left(\infty, \lambda_{i_{1}}\right), \ldots,\left(\infty, \lambda_{i_{p}}\right)\right\}
$$

and

$$
\sum_{j=1}^{p} \eta\left(\lambda_{i_{j}}\right)=\Theta \in Z \oplus\left(\bigoplus_{i=1}^{\infty} Z\right)
$$


where $\eta\left(\lambda_{i_{j}}\right)=D E G\left(A-b\left(\lambda_{i_{j}}+\varepsilon\right), D_{a}(Z)\right)-D E G\left(A-b\left(\lambda_{i_{j}}-\varepsilon\right), D_{a}(Z)\right)$ for sufficiently small $\alpha$.

Proof. - The proof of this theorem is the same as the proof of Theorem 4.1.

As an immediate consequence of Theorem 4.2 we obtain the following corollary.

COROLlaRY 4.3. - Under the hypotheses of Theorem 4.2, if moreover

1. function $b: R \rightarrow R$ is increasing in a neighbourbood of any point of the set $b^{-1}\left\{\mu_{1}, \ldots, \mu_{n}\right\}$ and $\left\{\mu_{1}, \ldots, \mu_{n}\right\} \cap\left\{\mu^{2}: \mu \in N^{*}\right\}=\emptyset$ or

2. function $b: R \rightarrow R$ is increasing in a neighbourbood of any point of the set $b^{-1}\left\{\mu_{1}, \ldots, \mu_{n}\right\}$ and for any $\mu_{i} \in\left\{\mu_{1}, \ldots, \mu_{n}\right\}$ there is a subrepresentation $R\left[1, k_{i}\right]$ of $\operatorname{ker}\left(A-\mu_{i}\right)$ which is not subrepresentation of $\operatorname{ker}\left(A-\mu_{j}\right)$ for any $\mu_{i} \neq \mu_{j}$

then for any $\lambda_{0} \in b^{-1}\left\{\mu_{1}, \ldots, \mu_{n}\right\} \mathcal{F}$ possesses an unbounded connected component $D\left(\lambda_{0}\right)$ which meets $\left(\infty, \lambda_{0}\right)$ (we add the points $\{(\infty, \lambda): \lambda \in R\}$ to dom $\left.(A) \times R\right)$. Moreover, if $\varepsilon_{1}>0$ is such that $\left[\lambda_{0}-\varepsilon_{1}, \lambda_{0}+\varepsilon_{1}\right] \cap b^{-1}\left(\left\{\mu_{1}, \ldots, \mu_{n}\right\}\right)=\left\{\lambda_{0}\right\}$ then for sufficiently large $\varepsilon_{2}>0$ and $M=\left\{u \in \operatorname{dom}(A):\|u\|>\varepsilon_{2}\right\} \times\left(\lambda_{0}-\varepsilon_{1}, \lambda_{0}+\varepsilon_{1}\right)$ either a) $D\left(\lambda_{0}\right)-M$ is bounded in $\operatorname{dom}(A) \times R$, in which case $D\left(\lambda_{0}\right)-M$ meets $\{0\} \times\left(Z^{\perp}\right)^{S^{1}} \times R \subset \operatorname{dom}(A) \times R$, or b) $D\left(\lambda_{0}\right)-M$ is unbounded in dom $(A) \times R$. If $\left.\mathrm{b}\right)$ occurs and $D\left(\lambda_{0}\right)-M$ bas a bounded projection on $R$, then $D\left(\lambda_{0}\right)-M$ meets $\left(\infty, \lambda_{0}^{\prime}\right)$, where $\lambda_{0} \neq \lambda_{0}^{\prime}$ and $b\left(\lambda_{0}^{\prime}\right) \in\left\{\mu_{1}, \ldots, \mu_{n}\right\}$. Additionally, $D\left(\lambda_{0}\right) \cap(\{0\} \times R) \neq \emptyset$.

REMARK 4.4. - Under the assumptions of Corollary 4.3 with «increasing» replaced by «decreasing» we still obtain the thesis of this corollary.

REMARK 4.5. - In order to study symmetry-breaking at infinity of periodic solutions of family (2.1) we can formulate corollaries which corresponds to Corollaries 3.5, 3.7, 3.8 .

\section{5. - Continuation of periodic solutions.}

In this section we study continuation of periodic solutions family (2.1). Throughout this section we assume that family (2.1) satisfies assumptions (F.1)-(F.3), (F.6), (F.7). In the following theorem we consider family of wave equations without resonance at the origin and at infinity (at level $\lambda=\lambda_{0}$ ). This theorem ensures the existence of unbounded connected set of solutions of family (2.1).

THEOREM 5.1. - Assume that

1. $\left\{\mu_{1}, \ldots, \mu_{n}\right\} \cap\left\{\mu^{2}: \mu \in N^{*}\right\}=\emptyset$,

2. $\mu_{0}, \mu_{\infty} \notin\left\{\mu_{1}, \ldots, \mu_{n}\right\}$,

3. $\left(\mu_{0}, \mu_{\infty}\right) \cap\left\{\mu_{1}, \ldots, \mu_{n}\right\} \neq \emptyset$. 
Then there exist $\alpha, \alpha^{\prime}, \beta>0$ such that there exist continua $\mathfrak{C}^{-}, \mathfrak{C}^{+}$with

$$
\begin{aligned}
& \mathcal{C}^{-} \subset \operatorname{dom}(A) \times\left(-\infty, \lambda_{0}\right] \subset\{(u, \lambda) \in \operatorname{dom}(A) \times R: A u=F(u, \lambda)\}, \\
& \mathcal{C}^{+} \subset \operatorname{dom}(A) \times\left[\lambda_{0},+\infty\right) \subset\{(u, \lambda) \in \operatorname{dom}(A) \times R: A u=F(u, \lambda)\},
\end{aligned}
$$

and for both $\mathcal{C}=\mathcal{C}^{-}$and $\mathcal{C}=\mathfrak{C}^{+}$the following statements are valid:

i) $\mathfrak{e} \cap\left(D_{\alpha}(Z)-c l\left(D_{\alpha^{\prime}}(Z)\right)\right) \times D_{\beta}\left(Z^{\perp}\right) \times\left\{\lambda_{0}\right\} \neq \emptyset$.

ii) $e$ is unbounded.

Proof. - By Lemma 2.8 the study of periodic solutions of family (2.1) is reduced to the study of critical points of a functional $a \in C^{2}(Z \times R, R)$. Since $\mu_{0}, \mu_{\infty} \notin\left\{\mu_{1}, \ldots, \mu_{n}\right\}$, $\nabla^{2} a\left(\infty, \lambda_{0}\right)$ and $\nabla^{2} a\left(0, \lambda_{0}\right)$ are isomorphisms. That is why we can fix $\alpha>\alpha^{\prime}>0$ such that $\nabla a\left(\cdot, \lambda_{0}\right)^{-1}(0) \cap\left(Z-D_{\alpha}(Z)\right)=\emptyset$ and $\nabla a\left(\cdot, \lambda_{0}\right)^{-1}(0) \cap D_{\alpha^{\prime}}(Z)=\{0\}$. Put $\Omega=D_{a}(Z)-$ $-c l\left(D_{\alpha^{\prime}}(Z)\right)$. By the properties of degree for $S^{1}$-equivariant gradient maps, see Theorem 3.9 of [23], we have

$\operatorname{DEG}\left(\nabla a\left(\cdot, \lambda_{0}\right), \Omega\right)=\operatorname{DEG}\left(\nabla a\left(\cdot, \lambda_{0}\right), D_{\alpha}(Z)\right)-\operatorname{DEG}\left(\nabla a\left(\cdot, \lambda_{0}\right), D_{\alpha^{\prime}}(Z)\right)=$

$$
=\operatorname{DEG}\left(\nabla^{2} a\left(0, \lambda_{0}\right), D_{a}(Z)\right)-D E G\left(\nabla^{2} a\left(\infty, \lambda_{0}\right), D_{\alpha^{\prime}}(Z)\right) \text {. }
$$

Taking into consideration the above and combining assumption 3 with Corollary 4.4 of [23] we obtain

$$
\operatorname{DEG}\left(\nabla a\left(\cdot, \lambda_{0}\right), \Omega\right) \neq \Theta \in Z \oplus\left(\bigoplus_{i=1}^{\infty} Z\right) \text {. }
$$

Since assumption $1, Z^{S^{1}}=\{0\}$ and consequently $\nabla a(0, \lambda)=0$ for all $\lambda \in R$. Applying Theorem 6.4 we prove that continua $C^{ \pm}$are unbounded. By the compactness of $c l\left(D_{\alpha}(Z)\right)$ and the continuity of a map $v$, given in Lemma 2.8, we can choose $\beta>0$ such that $v\left(c l\left(D_{\alpha}(Z)\right), \lambda_{0}\right) \subset D_{\beta}\left(Z^{\perp}\right)$. To complete the proof it is enough to define $\mathfrak{C}^{ \pm}=$ $=\left\{(z, v(z, \lambda), \lambda):(z, \lambda) \in C^{ \pm}\right\}$.

The following theorem describes connected set of solutions of family (2.1) which emanate from level $\lambda=\lambda_{0}$. We emphasize that in this theorem we consider family of wave equations with resonance at the origin, at level $\lambda=\lambda_{0}$.

THEOREM 5.2. - Assume that

1. $\left\{\mu_{1}, \ldots, \mu_{n}\right\} \cap\left\{\mu^{2}: \mu \in N^{*}\right\}=\emptyset$,

2. $\mu_{\infty} \notin\left\{\mu_{1}, \ldots, \mu_{n}\right\}$,

3. $\mu_{0} \in\left\{\mu_{1}, \ldots, \mu_{n}\right\}$,

4. if $\bigoplus_{i=1}^{n} \operatorname{ker}\left(A-\mu_{i}\right) \approx \bigoplus_{i=1} R\left[j_{i}, k_{i}\right]$ and $\operatorname{ker}\left(A-\mu_{0}\right) \approx \bigoplus_{i=1}^{n_{0}} R\left[j_{i}^{0}, k_{i}^{0}\right]$ then there is $k_{i_{0}} \in\left\{k_{1}, \ldots, k_{r}\right\}$ such that

(a) $k_{i_{0}} \neq \operatorname{gcd}\left\{\bar{k}_{1}, \ldots, \bar{k}_{p}\right\}$ for any $\bar{k}_{1}, \ldots, \bar{k}_{p} \in\left\{k_{1}^{0}, \ldots, k_{r_{0}}^{0}\right\}$,

(b) $m^{-}\left(\left(A-\mu_{0}\right)_{\mid R\left[j_{i 0}, k_{i 0}\right]}\right) \neq m^{-}\left(\left(A-\mu_{\infty}\right)_{\mid R\left[j_{i 0}, k_{i 0}\right]}\right)$. 
Then either $0 \in \operatorname{dom}(A)$ is not isolated in $\left\{u \in \operatorname{dom}(A): A u=F\left(u, \lambda_{0}\right)\right\}$ or there exist $\alpha, \alpha^{\prime}, \beta>0$ such that there exist continua $\mathfrak{C}^{-}, \mathfrak{C}^{+}$with

$$
\begin{aligned}
& \mathcal{C}^{-} \subset \operatorname{dom}(A) \times\left(-\infty, \lambda_{0}\right] \subset\{(u, \lambda) \in \operatorname{dom}(A) \times R: A u=F(u, \lambda)\}, \\
& \mathcal{C}^{+} \subset \operatorname{dom}(A) \times\left[\lambda_{0},+\infty\right) \subset\{(u, \lambda) \in \operatorname{dom}(A) \times R: A u=F(u, \lambda)\},
\end{aligned}
$$

and for both $\mathfrak{C}=\mathfrak{C}^{-}$and $\mathfrak{C}=\mathfrak{C}^{+}$the following statements are valid:

i) $\mathcal{C} \cap\left(D_{\alpha}(Z)-c l\left(D_{a^{\prime}}(Z)\right)\right) \times D_{\beta}\left(Z^{\perp}\right) \times\left\{\lambda_{0}\right\} \neq \emptyset$.

ii) $\mathcal{C}$ is unbounded.

PROOF. - The proof is in fact the same as the proof of Theorem 5.1. The only difficulty is that by assumption $3, \nabla^{2} a\left(0, \lambda_{0}\right)$ is not an isomorphism. Since $0 \in \operatorname{dom}(A)$ is isolated in $\left\{u \in \operatorname{dom}(A): A u=F\left(u, \lambda_{0}\right)\right\}$, we choose $\alpha>\alpha^{\prime}>0$ such that

$$
\nabla a\left(\cdot, \lambda_{0}\right)^{-1}(0) \cap\left(Z-D_{\alpha}(Z)\right)=\emptyset, \nabla a\left(\cdot, \lambda_{0}\right)^{-1}(0) \cap D_{\alpha^{\prime}}(Z)=\{0\}
$$

and put $\Omega=D_{\alpha}(Z)-c l\left(D_{a^{\prime}}(Z)\right)$. What is left is to show that $D E G\left(\nabla a\left(\cdot, \lambda_{0}\right), \Omega\right) \neq \Theta$. It is easy to check that a map $\nabla a\left(\cdot, \lambda_{0}\right)$ satisfies all the assumptions of Theorem 4.1 of [24]. Repeating the proof of this theorem we show that $\operatorname{DEG}\left(\nabla a\left(\cdot, \lambda_{0}\right), \Omega\right) \neq \Theta$, which completes the proof.

EXAMPLE 5.3. - In order to illustrate Theorem 5.2 we present here the following example. Let us consider family (2.1) satisfying all the assumptions of Theorem 5.2 with:

$$
11<\alpha<\mu_{\infty}<12<\mu_{0}=13<\beta<14, \quad T=2 \pi .
$$

It is clear that $\sigma(A) \cap(\alpha, \beta)=\{12,13\}, \operatorname{ker}(A-12) \approx R[1,2], \operatorname{ker}(A-13) \approx R[1,6]$. Letting $k_{i_{0}}=2$ we check that

1. $k_{i_{0}}=2 \neq \operatorname{gcd}\{6\}$,

2. $m^{-}\left(\left(A-\mu_{\infty}\right)_{\mid R[1,2]}\right)=0 \neq-2=m^{-}\left(\left(A-\mu_{0}\right)_{\mid R[1,2]}\right)$.

REMARK 5.4. - Notice that we cannot apply the Brouwer degree in order to prove Theorems 5.1, 5.2. From the Wang results proved in [32] we obtain $\operatorname{deg}\left(\nabla a\left(\cdot, \lambda_{0}\right)\right.$, $\Omega, 0)=0 \in Z$, where deg denotes the Brouwer degree.

The following theorem is analogous to Theorem 5.2. The only difficulty is that the eigenspace of $A$ which corresponds to eigenvalue $\mu_{0}$ contains trivial representation of the group $S^{1}$.

THEOREM 5.5. - Assume that

1. $\mu_{\infty} \notin\left\{\mu_{1}, \ldots, \mu_{n}\right\}$,

2. $\mu_{0} \in\left\{\mu_{1}, \ldots, \mu_{n}\right\}$,

3. $\mu_{0} \in\left\{\mu^{2}: \mu \in N^{*}\right\}$,

4. if $\bigoplus_{i=1}^{n} \operatorname{ker}\left(A-\mu_{i}\right) \approx R[k, 0] \oplus \bigoplus_{i=1}^{r} R\left[j_{i}, k_{i}\right] \quad$ and $\quad \operatorname{ker}\left(A-\mu_{0}\right) \approx R[1,0] \oplus$ 
$\bigoplus_{i=1}^{r_{0}} R\left[j_{i}^{0}, k_{i}^{0}\right]$ then there is $k_{i_{0}} \in\left\{k_{1}, \ldots, k_{r}\right\}$ such that

(a) $k_{i_{0}} \neq \operatorname{gcd}\left\{k_{1}, \ldots, k_{p}\right\}$ for any $k_{1}, \ldots, k_{p} \in\left\{k_{1}^{0}, \ldots, k_{r_{0}}^{0}\right\}$,

(b) $\gamma \cdot m^{-}\left(\left(A-\mu_{0}\right)_{\mid R\left[j_{i_{0}}, k_{i_{0}}\right]}\right) \neq m^{-}\left(\left(A-\mu_{\infty}\right)_{\mid R\left[j_{r_{0}}, k_{i_{0}}\right)}\right)$ for $\gamma=0,1$.

Then either $0 \in \operatorname{dom}(A)$ is not isolated in $\left\{u \in \operatorname{dom}(A): A u=F\left(u, \lambda_{0}\right)\right\}$ or there exist $\alpha, \alpha^{\prime}, \beta>0$ such that there exist continua $\mathfrak{C}^{-}, \mathfrak{C}^{+}$with

$$
\begin{aligned}
& \mathfrak{C}^{-} \subset \operatorname{dom}(A) \times\left(-\infty, \lambda_{0}\right] \subset\{(u, \lambda) \in \operatorname{dom}(A) \times R: A u=F(u, \lambda)\}, \\
& \mathfrak{e}^{+} \subset \operatorname{dom}(A) \times\left[\lambda_{0},+\infty\right) \subset\{(u, \lambda) \in \operatorname{dom}(A) \times R: A u=F(u, \lambda)\},
\end{aligned}
$$

and for both $\mathfrak{C}=\mathfrak{C}^{-}$and $\mathfrak{C}=\mathfrak{C}^{+}$the following statements are valid:

i) $e \cap\left(D_{a}(Z)-c l\left(D_{\alpha^{\prime}}(Z)\right)\right) \times D_{\beta}\left(Z^{\perp}\right) \times\left\{\lambda_{0}\right\} \neq \emptyset$,

ii) Either $\mathcal{C}$ is unbounded or else $\mathbb{C}\left(\operatorname{dom}(A)-\left(D_{a}(Z)-c l\left(D_{\alpha^{\prime}}(Z)\right)\right) \times D_{\beta}\left(Z^{\perp}\right)\right) \times\left\{\lambda_{0}\right\}$.

PROOF. - Repeat the proof of Theorem 5.2 using Theorem 4.2 of [24] instead of Theorem 4.1 of [24].

EXAMPLE 5.6. - In order to illustrate Theorem 5.5 we present here the following example. Let us consider family (2.1) satisfying all the assumptions of Theorem 5.5 with:

$$
48<\alpha<\mu_{0}=49<51<\mu_{\infty}<\beta<52, \quad T=2 \pi .
$$

It is clear that $\sigma(A) \cap(\alpha, \beta)=\{49,51\}, \quad \operatorname{ker}(A-49) \approx R[1,0] \oplus R[1,24], \quad \operatorname{ker}(A-51) \approx$ $\approx R[1,7] \oplus R[1,25]$. Letting $k_{i_{0}}=7$ we check that

1. $k_{i_{0}}=7 \neq \operatorname{gcd}\{24\}$,

2. $m^{-}\left(\left(A-\mu_{\infty}\right)_{\mid R[1,7]}\right)=2 \neq 0=m^{-}\left(\left(A-\mu_{0}\right)_{\mid R[1,7]}\right)$.

\section{6. - Appendix.}

In this appendix, for the conveniences of the reader, we present without proofs two global bifurcation theorems for $S^{1}$-equivariant gradient maps (an infinite-dimensional version of this theorem and outline of its proof one can find in [23], Theorem 5.6). Moreover, we recall a global bifurcation from infinity theorem for $S^{1}$-equivariant gradient maps (an infinite-dimensional version of this theorem and its proof one can find in [26], Theorem 2.11) and result about continuation of solutions. In fact in order to prove these theorems it is enough to repeat all the steps of the classical proofs replacing the Brouwer degree with the degree for $S^{1}$-equivariant gradient maps.

Let $Z$ be finite-dimensional representation of the group $S^{1}$. Assume that $a: Z \times R \rightarrow R$ is an $S^{1}$-equivariant $C^{2}$-function such that $0 \in Z$ is a critical point of $a(\cdot, \lambda)$ for any $\lambda \in R$ and for any real numbers $\lambda_{1}<\lambda_{2}$

$$
\#\left(\left\{\lambda \in R: \operatorname{det}\left(\nabla^{2} a(0, \lambda)\right)=0\right\} \cap\left[\lambda_{1}, \lambda_{2}\right]\right)<\infty .
$$


Let us fix $\lambda_{0} \in \mathfrak{Q}=\left\{\lambda \in R: \operatorname{det}\left(\nabla^{2} a(0, \lambda)\right)=0\right\}$ and $\varepsilon>0$ such that $\left[\lambda_{0}-\varepsilon, \lambda_{0}+\right.$ $+\varepsilon] \cap \mathfrak{Q}=\left\{\lambda_{0}\right\}$. Define bifurcation index $\eta\left(\lambda_{0}\right)$ by the formula

$$
\eta\left(\lambda_{0}\right)=D E G\left(\nabla a\left(\cdot, \lambda_{0}+\varepsilon\right), D_{\alpha}(Z)\right)-D E G\left(\nabla a\left(\cdot, \lambda_{0}-\varepsilon\right), D_{\alpha}(Z)\right),
$$

where $\alpha$ is sufficiently small. Let us denote by $C\left(\lambda_{0}\right)$ connected component of the set

$$
c l\{(v, \lambda) \in Z \times R: \nabla a(v, \lambda)=0 \text { and } v \neq 0\}
$$

such that $\left(0, \lambda_{0}\right) \in C\left(\lambda_{0}\right)$.

THEOREM 6.1. - Suppose that function $a: Z \times R \rightarrow R$ satisfies all the assumptions stated above, except of (6.1). Then, if $\eta\left(\lambda_{0}\right) \neq \Theta \in Z \oplus\left(\bigoplus_{i=1}^{\infty} Z\right)$ then

a) either component $C\left(\lambda_{0}\right)$ is unbounded in $Z \times R$,

b) or $\left(C\left(\lambda_{0}\right)-\left(0, \lambda_{0}\right)\right) \cap(\{0\} \times R) \neq \emptyset$.

THeOREM 6.2. - Suppose that function $a: Z \times R \rightarrow R$ satisfies all the assumptions stated above. Then, if $\eta\left(\lambda_{0}\right) \neq \Theta \in Z \oplus\left(\bigoplus_{i=1}^{\infty} Z\right)$ then

a) either component $C\left(\lambda_{0}\right)$ is unbounded in $Z \times R$,

b) or $C\left(\lambda_{0}\right) \cap(\{0\} \times R)=\{0\} \times\left\{\lambda_{i_{1}}, \ldots, \lambda_{i_{s}}\right\} \subset\{0\} \times \mathfrak{Q}$ and

$$
\sum_{j=1}^{s} \eta\left(\lambda_{i_{j}}\right)=\Theta \in Z \oplus\left(\bigoplus_{i=1}^{\infty} Z\right) \text {. }
$$

Let us consider $S^{1}$-equivariant $C^{2}$-function $a: Z \times R \rightarrow R$ such that

$$
\nabla a(v, \lambda)=[A-b(\lambda)](v)+\varphi(v, \lambda),
$$

where $A$ is a symmetric nondegenerate matrix and $\varphi(v, \lambda)=o(\|v\|)$, at $v=\infty$, uniformly on bounded $\lambda$-intervals. Put $\mathscr{F}=\{(v, \lambda) \in Z \times R: \nabla a(v, \lambda)=0\}$.

THEOREM 6.3. - If $\lambda_{0} \in \mathfrak{Q}=\{\lambda \in R: \operatorname{det}(A-b(\lambda))=0\}$ and $b(\lambda)-b\left(\lambda_{0}\right)$ changes sign at $\lambda_{0}$ then $\mathscr{T}$ possesses an unbounded connected component $Q\left(\lambda_{0}\right)$ which meets $\left(\infty, \lambda_{0}\right)$ (we add the points $\{(\infty, \lambda): \lambda \in R\}$ to $Z \times R)$. Moreover, if $\Theta \subset R$ is an open interval such that $\Theta \cap \mathfrak{Q}=\left\{\lambda_{0}\right\}$ and $\mathscr{K}$ is an $S^{1}$-invariant neigbbourbood of $\left(\infty, \lambda_{0}\right)$ whose projection on $R$ lies in $\Theta$ and whose projection on $Z$ is bounded away from 0 then either

1. $\mathscr{Q}\left(\lambda_{0}\right)-\mathscr{M}$ is bounded in $Z \times R$, in which case $\mathscr{O}\left(\lambda_{0}\right)-\mathfrak{M}$ meets $\mathscr{R}=$ $=\{(0, \lambda): \lambda \in R\}$, or

2. $\mathscr{O}\left(\lambda_{0}\right)-\mathscr{N}$ is unbounded.

If 2. occurs and $\mathcal{O}\left(\lambda_{0}\right)-\mathscr{M}$ has a bounded projection on $R$, then $\mathscr{O}\left(\lambda_{0}\right)-\mathscr{K}$ meets $\left(\infty, \lambda_{0}^{\prime}\right)$, where $\lambda_{0} \neq \lambda_{0}^{\prime} \in \mathcal{O}$. Additionally, if $\mathscr{O}\left(\lambda_{0}\right) \cap \mathcal{R}=\emptyset$ and $\#\{\lambda \in R$ : 
$\operatorname{det}(A-b(\lambda))=0\} \cap\left(\lambda_{1}, \lambda_{2}\right)<\infty$, for any real numbers $\lambda_{1}<\lambda_{2}$, then

and

$$
\mathscr{O}\left(\lambda_{0}\right) \cap\{(\infty, \lambda): \lambda \in R\}=\left\{\left(\infty, \lambda_{i_{1}}\right), \ldots,\left(\infty, \lambda_{i_{p}}\right)\right\}
$$

$$
\sum_{j=1}^{p} \eta\left(\lambda_{i j}\right)=\Theta \in Z \oplus\left(\bigoplus_{i=1}^{\infty} Z\right)
$$

where $\eta\left(\lambda_{i_{j}}\right)=D E G\left(A-b\left(\lambda_{i_{j}}+\varepsilon\right), D_{a}(Z)\right)-D E G\left(A-b\left(\lambda_{i_{j}}-\varepsilon\right), D_{a}(Z)\right)$.

From now on assume that $a: Z \times R \rightarrow R$ is an $S^{1}$ - equivariant $C^{2}$-function.

THEOREM 6.4. - Let there exist an open, bounded, $S^{1}$-invariant set $\Omega \subset Z$ and $\lambda_{0} \in R$ such that $\nabla a\left(\cdot, \lambda_{0}\right):(\Omega, \partial \Omega) \rightarrow(Z, Z-0)$ and that $D E G\left(\nabla a\left(\cdot, \lambda_{0}\right), \Omega\right) \neq \Theta \in Z \oplus\left(\bigoplus_{i=1}^{\infty} Z\right)$. Then there exists continua $C^{-}, C^{+}$with

$$
\begin{aligned}
& C^{-} \subset Z \times\left(-\infty, \lambda_{0}\right] \cap \nabla a^{-1}(0), \\
& C^{+} \subset Z \times\left[\lambda_{0},+\infty\right) \cap \nabla a^{-1}(0),
\end{aligned}
$$

and for both $C=C^{-}$and $C=C^{+}$the following statements are valid:

i) $C \cap \Omega \times\left\{\lambda_{0}\right\} \neq \emptyset$.

ii) Either $C$ is unbounded or else $C \cap(Z-\operatorname{cl}(\Omega)) \times\left\{\lambda_{0}\right\} \neq \emptyset$.

\section{REFERENCES}

[1] J. F. Adams, Lectures on Lie Groups, W. A. Benjamin Inc., New York-Amsterdam, 1969.

[2] H. Amann, Multiple Positive Fixed Points of Asymptotically Linear Maps, J. of Funct. Anal., 17 (1974), pp. 174-213.

[3] H. Amann, Saddle Points and Multiple Solutions of Differential Equations, Math. Z., 169 (1979), pp. 127-166.

[4] H. Amann - E. Zehnder, Nontrivial Solutions for a Class of Nonresonance Problems and Applications to Nonlinear Differential Equations, Ann. Sc. Norm. Super. Pisa, CC. Sci., IV, Ser 7 (1980), pp. 539-603.

[5] H. Amann - E. Zehnder, Periodic Solutions of Asymptotically Linear Hamiltonian Systems, Manuscr. Math., 32 (1980), pp. 149-189.

[6] H. Amann - E. Zehnder, Multiple Periodic Solutions for a Class of Nonlinear Autonomous Wave Equations, Houston J. of Math., 7 No. 2, (1981), pp. 147-174.

[7] T. BARTSCH, Topological Methods for Variational Problems with Symmetries, Lect. Notes in Math., 1560, Springer, 1993.

[8] J. Berkovits - V. Mustonen, An Extension of Leray-Schauder Degree and Applications to Nonlinear Wave Equations, Diff. and Int. Equat., 3 No. 5, (1990), pp. 945-963.

[9] R. BöHмE, Die Lösung der Verzweigungsgleichungen für nichtlineare Eigenwert-Probleme, Math. Z., 127 (1972), 105-126.

[10] H. Brezis, Periodic Solutions of Nonlinear Vibrating String and Duality Principles, Bull. Amer. Math. Soc., 8 (1983), pp. 409-426. 
[11] H. Brezis - J. M. CoRon - L. Nirenkerg, Free Vibrations for a Nonlinear Wave Equation and a Theorem of P. Rabinowitz, Comm. Pure Appl. Math., XXXIII (1980), pp. 667-689.

[12] K. C. Chang, Solutions of Asymptotically Linear Operator Equations via Morse Theory, Comm. Pure Appl. Math., 34 (1981), pp. 693-712.

[13] K. C. Chang, Infinite Dimensional Morse theory and Multiple Solution Problems, Progress in Nonlinear Differential Equations and Their Applications, 6, Birkhäuser Boston 1993.

[14] K. C. Chang - S. P. WU - S. LI, Multiple Periodic Solutions for Asymptotically Linear Wave Equation, Ind. Univ. Math. J., 31, No. 5, (1982), pp. 721-731.

[15] J. M. Coron, Periodic Solutions of a Nonlinear Wave Equation Without Assumption of Monotonicity, Math. Ann., 262 (1983), pp. 273-285.

[16] E. R. FAdell AND P. H. RaBinowitz, Generalized Cobomological Index Theories for Lie Group Actions with an Application to Bifurcation Questions for Hamiltonian Systems, Inv. Math., 45 (1978), pp. 139-174.

[17] K. Gȩ̧A, Degree for Gradient Equivariant Maps and Equivariant Conley Index, Topological Nonlinear Analysis, Degree, Singularity and Variations, Editors: M. Matzeu and A. Vignoli, Progress in Nonlinear Differential Equations and Their Applications, 27, Birkhäuser (1997), pp. 247-272.

[18] N. Hirano, On the Existence of Multiple Solutions for a Nonlinear Wave Equation, Journ. Diff. Equat., 71 No. 2, (1988), pp. 334-347.

[19] H. KIELHÖFER, Bifurcation of Periodic Solutions for Semilinear Wave Equations, Journ. Math. Anal. Appl., 68 (1979), pp. 408-420.

[20] H. Kielhöfer - P. Kótzner, Stable Periods of a Semilinear Wave Equation and Bifurcations of Periodic Solutions, J. Appl. Math. Phys. (ZAMP), 38 (1987), pp. 204-212.

[21] G. Mancins, Periodic Solutions of Some Semilinear Autonomous Wave Equations, Bollettino U. M. I. (5), 15 B (1978), pp. 649-672.

[22] P. H. Rabinowitz, Free Vibration for a Semilinear Wave Equation, Comm. Pure. Appl. Math., 31 (1978), pp. 31-68.

[23] S. RyBicki, $A$ Degree for $S^{1}$-equivariant Ortbogonal Maps and Its Applications to Bifurcation Theory, Nonlinear Analysis TMA, 23 No. 1, (1994), pp. 83-102.

[24] S. RyBICKI, Applications of Degree for $S^{1}$ - equivariant Gradient Maps to Variational Nonlinear Problems with $S^{1}$-symmetries, Topol. Meth. Nonlin. Anal., 9 (1997), pp. 383-417.

[25] S. RYвICKI, On Rabinowitz Alternative for the Laplace-Beltrami operator on $S^{n-1}$, Diff. and Int. Equat, 9 No. 6, (1996), pp. 1267-1277.

[26] S. RYвіскI, On Bifurcation From Infinity for $S^{1}$-equivariant Potential Operators, Nonlinear Analysis TMA, 31 No. $3 / 4$ (1998), pp. 343-361.

[27] S. RYBICKI, Global Bifurcation of Solutions of Elliptic Differential Equations, Journ. of Math. Anal. and Appl., 217 (1998), pp. 115-128.

[28] S. RyBicki, On Periodic Solutions of Autonomous Hamiltonian Systems via Degree for $S^{1}$-Equivariant Gradient Maps, Nonlinear Analysis TMA, 34 (1998), pp. 537-569.

[29] F. TAKENS, Some remarks on the Böbme-Berger Bifurcation Theorem, Math Z., 12591972 ), 359-364.

[30] Z. Q. WANG, Symmetries, Morse Polynomials and Applications to Bifurcation Problems, Acta. Mathematica Sinica, New Series, 6 No. 2, (1990), pp. 165-177.

[31] Z. Q. WANG, Equivariant Morse Theory for Isolated Critical Orbits and Its Applications to Nonlinear Problem, Lect. Notes in Math., 1306 Springer (1988), pp. 202-221.

[32] Z. Q. WANG, Symmetries and the Calculations of Degree, Chi. Ann. of. Math., 10 B (1989), pp. 520-536. 